\title{
REVIEWS
}

\section{Psychiatric Aspects of Herpes Simplex Encephalitis, Tick-Borne Encephalitis and Herpes Zoster Encephalitis Among Immunocompetent Patients}

Division of Consultation Psychiatry and Neuroscience, Department of Psychiatry, Wroclaw Medical University, Poland

A - research concept and design; $\mathbf{B}$ - collection and/or assembly of data; $\mathbf{C}$ - data analysis and interpretation;

$\mathbf{D}$ - writing the article; $\mathbf{E}$ - critical revision of the article; $\mathbf{F}$ - final approval of article; $\mathbf{G}$ - other

\begin{abstract}
The psychopathological symptoms occurring in the course of diseases associated with infections are often initially isolated and non-characteristic, and may cause diagnostic difficulties. Moreover, such disorders tend to be less responsive to psychiatric management. Among possible causes such as trauma, neoplasm and vascular changes, inflammatory changes of the brain as a result of a viral infection should also be considered. There were 452 registered cases of viral encephalitis in Poland in 2010, and although not very prevalent they remain a severe and lifethreatening condition. What is more, the frequently occurring neurological and psychiatric complications of viral encephalitis often result in permanent disabilities, causing a significant decrease in the quality of life. This article presents the three types of encephalitis that are most prevalent among immunocompetent patients in Poland, i.e. herpes simplex encephalitis (HSE), tick-borne encephalitis (TBE) and herpes zoster encephalitis (HZE). The psychopathology of the acute phase of the infection, the residual symptoms, features apparent in imaging studies and some neuropathological aspects are also presented. The paper also focuses on psychiatric aspects of the diagnostics and treatment of the described conditions. The clinical pictures of these infections are quite specific, although they cover a wide range of symptoms, and these characteristic features are described. The aim of this review is also to show the significance of thorough diagnostics and a multidisciplinary approach to patients with viral CNS infections (Adv Clin Exp Med 2015, 24, 2, 361-371).
\end{abstract}

Key words: herpes simplex encephalitis, varicella zoster encephalitis, tick-borne encephalitis, mental disorders.

There were 452 registered cases of viral encephalitis in Poland in 2010 and although not very prevalent it remains a severe and life-threatening condition [1]. Moreover, the frequently occurring neurological and psychiatric complications often result in permanent disabilities, causing a significant decrease in the patient's quality of life [2]. There are over 100 known viral causative agents of CNS infections [3]. Encephalitis may be sporadic or epidemic with regard to etiology and depending on whether it is acute, subacute, progressive or chronic $[2,3]$. Herpes simplex virus $1(\mathrm{HSV}-1)$ is the etiologic agent in $20-52 \%$ of the cases of acute sporadic encephalitis. Ebstein-Barr, Varicella-Zoster, rabies and West Nile viruses are less frequent agents. Arboviruses, enteroviruses and the mumps virus can cause acute epidemic encephalitis $[3,4]$. However, in most cases (60\%) attempts to identify the pathogen causing the disease are in vain [3]. Cytomegalovirus, Ebstein-Barr virus and human herpes virus 6 occur more often as causative agents of encephalitis in the context of immunocompromise caused by human immunodeficiency virus (HIV) infection and post-transplant treatment [5].

According to Mumenthaler et al., disorders of consciousness (apparent in $97 \%$ of viral encephalitis patients), fever (87\%), personality changes $(81 \%)$, headaches $(79 \%)$, aphasia $(72 \%)$, seizures (42\%) and hemiparesis (35\%) are the typical symptoms of viral encephalitis [6]. It is always worth 
considering, however, that the clinical manifestations of many acute and chronic viral infections, as well as their complications, include a wide variety of mental disorders $[2,3,7]$. The etiology of these disorders often consists of multiple factors. Inflammatory, degenerative and necrotic pathomorphological changes inflicted directly by the viruses on the central nervous system can the occurrence of mental disorders [4]. Elevated proinflammatory cytokine levels also have an adverse effect on the patients' mental condition [8]. Psychopathological symptoms occurring in the course of viral infections pose diagnostic difficulties. Specifically, herpes simplex encephalitis (HSE) accompanied by a rapidly developing psychosis can be misdiagnosed as an episode of schizophrenia, while the infectious background of the psychopathology requires immediate antiviral treatment $[9,10]$.

This article presents the 3 types of encephalitis that are prevalent among immunocompetent patients - i.e., herpes simplex encephalitis (HSE), tickborne encephalitis (TBE) and herpes zoster encephalitis (HZE) [1, 11]. The aim of this review is also to show the significance of thorough diagnostics and a multidisciplinary approach to patients with viral CNS infections.

\section{HSV}

With regard to the serotypes of HSV, various authors concur that $90 \%$ of encephalitis cases in patients with a healthy immune system are caused by HSV1, whereas HSV2 tends to cause aseptic meningitis or encephalitis in immunocompromised people and newborns [3, 13-16]. There are two peaks of morbidity in terms of the patients' age: one under the age of 20 and the other in the mid-sixties [17]. The prevalence of HSE varies between 1/250,000 to $1 / 500,000$, with one-third of the patients under the age of 20 [16]. According to Steiner, HSE is not a feature of a person with a compromised immune system, with the exception of acquired immunodeficiency syndrome (AIDS) and after a bone marrow transplant [12]. Nevertheless, patients in immunosuppression are at risk of a less favorable and atypical course of the infection [13]. On the other hand, typical symptoms of encephalitis, such as headaches or fever, may be absent in patients with AIDS. In these cases, the only manifestation of HSE may be a disturbing change in behavior [12].

\section{Neuropathology}

It has still not been definitely established whether HSE develops from a latent or primary infection [12]. It is estimated that about $30 \%$ of HSE cases are the effect of a primary infection, while other cases are due to a reactivation of the virus $[9,16,18]$. There are three hypotheses concerning the mechanisms of HSV brain entry $[12,17]$. The first one assumes that HSV 1 reactivates from its reservoir of latency - the trigeminal ganglion - and spreads into the frontal and temporal lobes through the trigeminal nerve. It has been also proven (not only in animal experimental models) that the olfactory tract constitutes a probable route of viral entry [17]. The virus has in some cases been identified in brain tissue, which suggests that it could reactivate directly in the CNS. Finally, it has not been excluded that HSE is a result of a primary infection. According to some authors, in nearly half the cases, the virus that caused HSE was a different strain from the one causing cold sores in the same patients, which supports the primary-infection hypothesis $[12,17]$. Autophagy inhibition and the fact that the infected neurons have disrupted major histocompatability complex (MHC) antigen expression have been proposed as mechanisms facilitating HSV1 evasion of the host's immune response [17].

Neuropathologically HSE manifests as acute necrotic encephalitis, more pronounced in the cortex than in the white matter [19]. The fronto-orbital region, temporal lobes, hippocampus, cingulate gyrus and insular cortex are the most affected areas. In these sites brain edema followed by hemorrhagic necrosis precedes the cytolysis of neurons, astrocytes and oligodendrocytes $[3,10,15,20]$. The relative proximity of the nasal cavity (the probable site of the virus infiltration) has been proposed as an explanation of the selective frontal and temporal lobe involvement $[12,17]$. A specific HSV affinity to the limbic cortex has also been postulated [18]. The location of the inflammatory and necrotic changes explains the behavioral, emotional and personality disorders occurring in the course of HSE [20]. The control of regional immune and inflammatory mechanisms by a complex network of cytokines has been suggested by Skoldenberg. Furthermore, the intrathecal cellular and humoral activation may last beyond acute HSE [21].

\section{Symptoms of the Acute Phase}

The most frequent prodromal HSE symptoms include headaches, joint and muscle pain, nausea and vomiting, psychomotor retardation, confusion and irritability [4-6]. Focal, partial or generalized seizures and sometimes meningism are the usual neurological symptoms $[9,16]$. The focal symptoms are associated with the pathology of temporal 
lobes and orbitofrontal structures, and they include anosmia, olfactory and gustatory hallucinations or memory impairment with undisturbed intellect [19]. Coma is often preceded by a prominent delirious phase [5]. Cases of initially subacute HSE that progress over a period of several months are reported as rare occurrences [3].

Diagnostic difficulties may be encountered when a paranoid syndrome with delusions, hallucinations (mostly acoustic), psychomotor agitation, anxiety and aggression occurs as an initial manifestation of HSE. It tends to progress into a catatonic stupor with mutism $[9,10,14,16]$. The patient is usually placed under psychiatric care until the organic etiology of the symptoms is suggested by neurological abnormalities like upper motor neuron lesion, aphasia or paresis $[9,14,16]$.

\section{Diagnostics}

Patients who have pronounced psychopathological symptoms with minor abnormalities in diagnostic tests and a lack of meningism or other signs that might suggest a viral infection are the most difficult to diagnose $[10,14]$. A high degree of suspicion is necessary when an atypical clinical picture is present. A misleading set of symptoms is estimated to be present in as many as $20 \%$ of cases [5]. It might be confusingly similar to an acute psychotic syndrome, psychoneurosis (conversion disorders with fits), delusional disorder or mania $[5,9,16]$. The course of these symptoms is usually dramatic. An acute adverse reaction to neuroleptics may also indicate an organic cause of the symptoms $[9,16]$.

The polymerase chain reaction (PCR) for the detection of viral DNA in the cerebrospinal fluid (CSF) is the gold standard for HSE diagnostics [5]. The best HSV-PCR assay results are considered to be obtainable 2-10 days from the disease onset [13]. Magnetic resonance remains the most sensitive diagnostic method for the detection of early changes in the course of HSE. Signal disturbances are observed in the medial parts of the temporal lobes, spreading towards the insular cortex and the lateral parts of the basal nuclei $[15,16]$. Changes in the cingulate gyrus and the contralateral temporal lobe in magnetic resonance imaging (MRI) are highly suggestive of HSE. The EEG presentation of HSE is non-specific and may involve slowing or lateralizing epileptiform temporal discharges [5]. On the first days after the manifestation of symptoms, computed tomography might not show any abnormalities. Later on, extensive hypodense areas are visible in the temporal lobes $[10,15]$. Although the results often exhibit lymphocytic pleocytosis and elevated protein levels, $5-10 \%$ of CSF samples do not show abnormalities and the standard examination is considered neither characteristic nor diagnostic [13].

The prognosis in HSE is poor unless antiviral therapy is undertaken immediately after the appearance of the first symptoms $[3,12,19]$. After the introduction of acyclovir to HSE treatment, the original death rate of $70 \%$ decreased to 20-30\% [17]. Factors like age under 30, HSE duration of less than 4 days and a consciousness rating of more than 6 on the Glasgow Coma Scale at the beginning of acyclovir treatment correlate with a better outcome [15]. Foscarnet has been reported to be an alternative in immunocompromised patients who may prove resistant to acyclovir treatment [17].

\section{Sequelae}

At least $80 \%$ of HSE survivors are considered to suffer from persisting cognitive impairments or other neuropsychiatric disorders [3]. It has been estimated that the risk of cognitive deficits occurring in the course of HSE is 2 to 4 times higher than in other neuroinfections [4]. These disorders include severe deficits in anterograde memory (such as Korsakoffs syndrome) and also retrograde memory (less severe), aphasia, visuospatial and executive dysfunctions [3, 22]. From 25\% to $75 \%$ of patients are reported to suffer from dense anterograde amnesia [7]. Concomitant retrograde amnesia tends to be less frequent, although sometimes it is present in isolation $[5,22]$. Children tend to develop mental retardation, whereas adults exhibit signs of early dementia [5]. The period of transient encephalitic amnesia is the best predictor of future cognitive functioning [23]. HSV1 is known to inflict preferential damage to the temporal and orbitofrontal cortex, which is associated with cognitive disorders $[4,19]$. These changes are bilateral but not symmetric [15]. A more affected left hemisphere results in more pronounced verbal memory and language disorders, while the involvement of the right hemisphere does not cause much functional impairment [17]. There have also been reports of HSE cases with signs of autism in their course. Although some patients recovered fully after antiviral treatment, some did not; and even after the acute phase of the infection, the autism progressed. A characteristic triad of symptoms was present in those patients, including social functioning disorders, verbal and nonverbal communication disorders and stereotyped patterns of behavior [18]. Moreover, hemiparesis, epilepsy, affective and behavioral disorders are 
noted among the sequelae of HSE. There are several reports of category-specific anomia and more severe disorders of speech [5]. There is also evidence that hypersexuality, hyperphagia, prosopagnosia and hyperoralism, which are typical of the Klüver-Bucys syndrome, may occur [3].

According to a study by McGrath et al., memory impairment (short-term in particular) was noted in $69 \%$ out of 34 HSE survivors, anosmia in $65 \%$, personality and behavior disorders in $45 \%$, speech impairment in $41 \%$ and epilepsy in $24 \%$ [24]. Eachut et al. described a patient whose symptoms after HSE resembled fronto-temporal dementia. Disorders of speech, writing, cognitive functions and orientation were present, as well as affect liability and frontal lobe behavioral disorder (disinhibition, hyperoralism, object grabbing) [20]. According to those researchers, it is difficult to state how the cognitive functions are affected by the infection itself and to what extent the virus induces an independent neurodegenerative process [20]. Among HSE survivors there is a high prevalence (up to $45 \%$ ) of psychiatric disorders that occur as much as several years after the acute condition. They include behavior and personality disorders, and also psychoses and emotional liability [5]. The relationship between conditions like Alzheimer's disease (AD), multiple sclerosis (MS) and acute/chronic/reactivated HSV infection constitutes an interesting field for research. There is evidence that in some cases the pathogenesis of $\mathrm{AD}$ an MS may be associated with an HSV infection. The pathology of both Alzheimer's disease and HSE affects the limbic system [17]. According to Itzhaki et al., the presence of HSV1 in the brain parenchyma contributes to multiplying the risk of $\mathrm{AD}$ development in subjects that carry the APOE $\varepsilon 4$ allele [25].

\section{TBE Virus}

In several European countries, including Poland, TBE remains one of the leading causes of CNS infections [8]. It is believed that in Poland its prevalence is equal to that of HSV, and in 2012 it even made up as much as $65 \%$ of the registered viral encephalitis cases $[1,3,12]$. Central and Eastern Europe, the Scandinavian Peninsula, Asia and the Far East are the endemic territories of TBEV [26]. Three types of the virus may be distinguished: the European, Siberian and Far Eastern. Ixodes ricinus is considered the vector for the first one and ixodes persulcatus for the latter two [27]. During the tick bite, the tick's saliva transmits the infection. It is also possible to acquire the virus through unpasteurized milk [26]. TBE should be considered in the differential diagnostics even in regions outside of the known endemic territories, due to the fact that recent years have brought not only an increase in TBE prevalence but also outbreaks of the disease in new areas [28]. Langerhans cells, which are dendritic skin cells, are the inoculation site in which the virus initially replicates before being transported to the local lymph nodes. Then the virus infects the $T$ and B lymphocytes, as well as macrophages of the lymph nodes, spleen and thymus [26]. The high production and consequent viral load of the primarily infected organs contributes to the transmission of the virus through the blood/brain barrier. The endothelial cells are the next replication site from which TBEV invades the brain directly [27].

\section{Symptoms}

The TBE incubation period lasts from 4 to 28 days. The tick bite remains unnoticed by nearly $1 / 3$ of the patients $[8,26,27]$. A biphasic disease with prodromal symptoms is observed in $75 \%$ of the cases [26]. The first phase of the disease (lasting 2-7 days) consists of influenza-like symptoms: fever, headaches, muscle and joint pains, inflammation of the upper respiratory tract and neurasthenia. However, patients also report mental and physical fatigue, irritability, concentration disorders, sleep disorders, short term memory and appetite disorders, while the symptoms of encephalitis are absent $[29,30]$. After a few days of relative remission, the illness proceeds to the second phase, manifested as meningitis in $50 \%$ of the cases, meningoencephalitis in $40 \%$ and meningoencephalomyelitis in 10\% [26]. The characteristic symptoms of meningitis are usually present, although they may not be prominent. Meningitis is often accompanied by headache, vertigo, nausea, vomiting and high fever $[8,26]$. The neurological signs of encephalitis include convulsions, paralysis, involuntary movements, epilepsy with partial constant seizures and cranial nerve palsy. Various mental disorders, particularly qualitative and quantitative consciousness disorders (usually without coma) and also retrograde amnesia may occur $[29,30]$. Seizures are not a frequent occurrence, but $1 / 3$ of the patients may develop altered consciousness. In a study by Kaiser, $12 \%$ of the patients scored under 7 points on the Glasgow Coma Scale; among other symptoms, restlessness, increased movements of the face and limb muscles, tremors of the tongue, vertigo and speech disorders were observed [26]. When the cranial nerves are involved, symptoms connected with the function of the ocular, facial and pharyngeal muscles are the most common [27]. There is also a group of patients who develop 
delirium or psychosis [26, 29]. Ataxia followed by paresis or paralysis of one or more limbs is considered the most characteristic feature of TBE, not only in human patients but also in monkeys infected during experiments [8]. The TBE virus exhibits a predilection for certain brain areas, which explains the occurrence of these and other neurologic symptoms [8]. The acute phase of the disease is sporadically associated with the occurrence of depression, catatonia, delusions, cognitive impairment, neurosis and insomnia. The typical neurological and systemic symptoms may be preceded by the psychopathological signs, thus augmenting the diagnostic difficulties [29-31]. Juchnowicz et al. reported the presence of mental disorders in $60.8 \%$ of TBE patients in the first and second weeks of the disease duration. Cognitive deficits accounted for more than $75 \%$ of those disorders. Most of the remaining patients had organic depressive disorders. In most cases the cognitive deficits affected the visual, direct and permanent memory, learning skills and concentration ability [30]. Although the mortality rate of TBE reaches no higher than $2 \%$, the prevalence of neurological and psychiatric complications is estimated at $28.55 \%[29,31]$.

\section{Post-Encephalitic Syndrome}

About $35 \%$ to $58 \%$ of patients have been reported to suffer from post-encephalitic TBE syndrome, which causes a long-term condition that contributes to a lower quality of life [31]. The symptoms that have most commonly been reported include lower stress tolerance, memory impairment, balance disorders, headaches, dysphasia, impaired hearing and occasionally spinal paralysis [26]. In the second part of the Juchnowicz et al. study, the same group of patients was reevaluated 3 months after the regression of clinical symptoms, with CSF abnormalities as well as their mental status taken into consideration. The prevalence of mental disorders increased to $72.5 \%$ and organic depressive disorders became more common than cognitive impairment. The severity of TBE was correlated with higher incidence and severity of organic depressive disorders in the period of convalescence. The authors found no similar correlation with regard to the occurrence of mental disorders in the acute phase of the disease. Among the stated psychopathological symptoms, attention deficits $(72.1 \%$ of the patients), slowness of thought (69.8\%), learning impairment (60.5\%), depressed mood (60.5\%), emotional liability $(46.5 \%)$ and mutism (41.9\%) were the most frequent [30]. TBE leaves a predisposition to dementia. Behavioral disorders such as irritability or lack of consistency in action may indicate its onset [31]. A recent study by Fowler et al. that included an evaluation of long-term TBE outcomes in 55 children gave evidence of the risk of incomplete recovery from TBE. The psychological assessment of children was conducted within 2-7 year post-TBE. Residual difficulties were reported in as many as $2 / 3$ of the cases. The complaints particularly regarded cognitive functions, irritability, fatigue and headaches. In more than $1 / 3$ of the subjects executive functioning was impaired, especially in initiating and organizing activities and working memory [32]. Mental disorders are an important part of TBE clinical symptoms not only in the prodromal and acute phase of the infection but also for many years afterwards. This may suggest a sub-clinically active inflammatory process resulting in progressive CNS damage [30, 31]. Persistent cognitive impairment and depressive disorders are sequelae of TBE that lead to a significant decrease in the patients' quality of life [26, 31].

\section{Neuropathology}

Diffuse lymphocytic infiltrations in the meninges, particularly of the cerebellum, have been demonstrated in post-mortem studies of the brains and medullas of monkey models as well as patients that died due to TBE [26]. Microscopic lesions and edema were prominent features observed throughout the CNS, especially in the grey matter, medulla oblongata, pons, cerebellum, brainstem, basal ganglia, thalamus and spinal cord [26, 27]. Lymphocytic perivascular infiltrations, gliosis and nerve cell necrosis were the apparent pathological changes [8]. The motor cortex is the only cortical site affected. The pathologies present include degeneration of pyramidal cells with necrosis and superficial lymphocytic aggregations with gliosis [26]. Lesions are a particularly common finding among the cerebellar purkinje cells and the anterior horn cells of the spinal cord. Infiltrates consisting of lymphocytes and neuronal rarefaction have also been reported in the mesencephalon and diencephalon [26].

\section{Diagnostics and Treatment}

During the second phase of the disease, when the CNS symptoms become prominent, the diagnostics are straightforward. TBEV-immunoglobulin $M(\operatorname{IgM})$ are certain to be detected in the serum samples, and usually TBEV-IgG antibodies as well [8]. Before seroconversion, RT-PCR to isolate TBEV-RNA from blood samples is the method 
of choice. At the onset of the second phase and in cases of a progressive course of the infection, the TBEV may occasionally be detected in the CSF [27]. On the other hand, there is a risk of false positive results due to the nonspecific character of the antibodies used in the ELISA methods. The molecules may cross-react with other flaviviruses, hence in populations at risk of exposure to other pathogenic flaviviruses, a neutralization assay is required to confirm the results [8]. CSF samples obtained in the course of meningoencephalitis usually reveal pleocytosis, normal glucose and lactate levels, with the level of proteins (the CSF/serum albumin ratio) increased but not very high [8]. Although MRI studies of TBE patients may reveal brain damage, it remains a rare finding; such evidence was found in no more than $18 \%$ of the patients examined in a study involving 102 TBE patients [8]. The abnormalities mainly regarded the thalamus and occasionally the cerebellum, brainstem, nucleus caudatus and the basal ganglia [8,27]. Although $77 \%$ of the EEG assays exhibit anomalies, like MRIs they are not specific and have little diagnostic value [27].

There is no specific treatment for tick-borne encephalitis $[27,29,30]$. With regard to the treatment of the progressive disease, study results suggest a distinction between two types. The first one is characterized by an absence of TBEV and tends to be susceptible to immunomodulatory treatment, while the other one features detectable TBEV and tends to be responsive to antivirals [27]. Active immunization provides effective prevention against TBE. It is recommended that in the endemic territories not only farmers and rangers, but also tourists and local residents should be vaccinated. Three doses of the inactivated virus are administered on a 0 -3-12-month schedule (an accelerated schedule of $0-1-3$ months is also possible) [1].

\section{Varicella Zoster Virus}

Depending on the immune status (primary infection or not), the VZV is the causative agent of chickenpox or herpes zoster (HZ) [7, 33]. A primary infection leaves the virus latent in the trigeminal and thoracic ganglia, where the presence of VZV DNA is detected by PCR and in situ hybridization techniques [34]. The reactivation and replication of the VZV is initiated during states of decreased immunity, particularly decreased cellular immunity, when it migrates from the ganglia along the neuronal axons to the skin, resulting in herpes zoster [33]. On rare occasions, the infection may generalize secondarily and cause encephalitis [34]. HZ is more characteristic of middle-aged and older patients, and patients with a compromised immune system are at risk of a more severe course [33]. Only $5 \%$ of patients hospitalized due to $\mathrm{HZ}$ and no more than $0.05 \%$ of adult patients with chickenpox tend to develop herpes zoster encephalitis (HZE) [7]. However, although it is rare, it is a severe and life threatening complication of VZV infection. On the other hand, in terms of the etiological agents of viral encephalitis, VZV is not uncommon. According to the results of a large Finnish study involving 3000 subjects with encephalitic symptoms, VZV turned out to be the most prevalent causative agent associated with viral encephalitis (3 times more than HSV) [11]. Another study on encephalitis among children (also in Finland) consistently found that $25 \%$ of all cases were due to VZV infection. In a retrospective study in England, HZE proved to be the second most prevalent (13\%) viral encephalitis [2].

\section{Neuropathology}

HZE neurological symptomatology is generally associated with the infection of both small and large cerebral arteries. It causes a vasculopathy that results in uni- or multifocal brain tissue infarctions [34]. Three types of brain damage may be present. Ischemic or hemorrhagic infarctions may accompany the large/medium vessel vasculopathy, resulting in vasculitis, arteritis and hemiparesis [7]. Large vessel unifocal granulomatous arteritis may proceed without any skin lesions, or it may be comorbid with chickenpox/HZ. It tends to occur in patients with a competent immune system, with a mortality rate reaching $25 \%$ [17]. Small vessel vasculopathy, which is characteristic of patients with compromised immunity, causes not only ischemic but also demyelinating lesions and contributes to the development of multifocal leukoencephalopathy $[7,34]$. It is uncommon for skin eruptions to occur simultaneously with this type of vasculopathy [17]. Ventriculitis or periventriculitis is the third possible type of brain damage due to VZV infection [7, 34].

\section{Symptoms}

It usually takes several weeks for typical encephalitis to develop. The acute stage is associated with the occurrence of cognitive deficits with confusion and somnolence $[7,33]$. The profile of the cognitive deficits present includes worse verbal and visual reasoning, memory and attention impairment, perseverations and deficits in planning and impulse control [5]. Although hallucinations 
are reported to be a frequent occurrence, they are considered to be an effect of delirium or high fever. Perception disorders may also result from the posterior temporal ictal activity during seizures $[5,7]$. Psychosis and mood liability from euphoria to dysphoria are not only features of the acute stage, but may also persist as components of the residual symptoms [22]. A brief course of steroids during acute encephalitis may be a supportive addition to the standard 7-10 day acyclovir treatment [34].

\section{Prognosis}

Currently, HZE is rarely fatal, and most survivors eventually achieve their prior cognitive status [7]. Nonetheless, persisting and irreversible deficits like memory impairment, dysphasia and even dementia may occur [4, 33]. Based on their observations of HZE recoveries, McKenna et al. reported deficits in attention, concentration, planning, impulse control and insight [35]. Hokkanen et al. conducted a study among immunocompetent patients recovering from encephalitis (including HZE) that had been treated with acyclovir; they reported that in comparison with the controls, the HZE subjects had decreased performance IQ, impaired visual memory and were slower at the Stroop test tasks. However, in comparison with HSE survivors, aphasia, agnosia and apraxia were absent, and the present memory deficits were less severe. Other observed conditions included slowing of thought, behavioral disinhibition in the form of impulsiveness and verbosity, euphoria or depression and emotional liability. It is noteworthy that all of the subjects eventually returned to occupational activity, as the intensity of these disorders showed a tendency to decline and subside with time [23]. On the other hand, not all authors concur regarding the relatively benign character of the neuropsychological deficits associated with HZE. In a case report by Bangen et al., a previously healthy, immunocompetent and correctly treated patient developed persisting verbal and visual memory impairment with hippocampus volume decrease [33]. The presence of slowness of thought, forgetfulness, mood and behavior liabilities while the higher nervous functions remained intact suggests that in the course of HZE a subcortical dysfunction occurs [7].

\section{Diagnostics}

VZV vasculopathy may be difficult to diagnose when the characteristic skin changes remain absent or their occurrence is separated by a considerable period of time. Focal ischemic or hemorrhagic lesions are usually present in CT or MRI brain scans. Arterial abnormalities are observed during angiography. Mononuclear pleocytosis is detectable in $2 / 3$ of CSF samples [36]. As in the diagnostics of HSV encephalitis, CSF-PCR for VZV-DNA remains the method of choice [13]. On the other hand, the detection of CSF antibodies against VZV is, as Nagel et al. stated, of more diagnostic value than PCR, which may not be sensitive enough $[36,37]$. Moreover, characteristic Cowdry A inclusion bodies may be observable in pathologic samples, which can also be examined with the use of in situ hybridization [34].

\section{Management in Cases of Suspected and Confirmed Viral Encephalitis in Adults}

Although the presentation of exact algorithms for treating encephalitis is not the subject of this review, some basic information that should facilitate emergency management seems worth including. Diagnostic procedures for encephalitis should be conducted when febrile symptoms, rash, new focal neurological signs or a new onset of seizures is accompanied or succeeded by psychiatric signs such as changes in behavior, personality or cognition (the Mini Mental State Examination might be useful), speech disturbances, consciousness disorders or disorientation [38]. Hyponatremia may occur in routine laboratory tests, since the syndrome of inappropriate antidiuretic hormone (SIADH) is often comorbid with encephalitis [38, 39]. A lumbar puncture (LP) must be conducted as soon as possible in all cases of patients that lack contraindications. A CT preceding the LP is indicated for patients with conditions such as moderate to severe impairment of consciousness (reduced or fluctuating GCS < 13), focal neurological signs, papilloedema, relative bradycardia with hypertension, systemic shock or impaired immunity [38]. A standard CSF examination (opening pressure, morphology, protein and glucose) should be followed by a CSF PCR test for HSV 1 and 2, VZV and enteroviruses [40]. If the CSF was not tested for viral DNA/RNA during the acute phase of the illness (10-14 days after the first signs), the CSF and blood plasma should be tested for HSV IgG antibody. Every patient suspected of encephalitis should be tested for HIV [38]. An MRI examination of the brain is indicated as soon as possible (ideally within $48 \mathrm{~h}$ ) in all cases of patients with uncertain diagnoses. An EEG is not a routine study; it is indicated for patients with subtle 
motor or non-convulsive seizures, or with behavioral changes of uncertain etiology [38]. Intravenous aciclovir (10 mg/kg 3 times daily) is administered when the results of the CSF examination and/or imaging studies suggest encephalitis or are unavailable within $6 \mathrm{~h}$ post admission, or when the patient's state is severe or deteriorating. This treatment is also administered in every case of a clinical suspicion of HSV/VZV encephalitis, even when the results of additional tests do not show abnormalities [38]. Intravenous aciclovir treatment lasts for at least 14-21 days and is maintained until the CSF HSV PCR changes to negative $[38,39]$. In cases of VZV encephalitis with a pronounced vasculitic component, corticosteroids are administered along with the aciclovir [40]. Post-encephalitic syndrome (described above) appears to be a separate therapeutic challenge co-occurring in some encephalitis cases. Several specialists are often engaged in the management of persisting emotional, cognitive and behavioral disorders. The importance of comprehensive neuropsychological treatment cannot be overestimated; it consists of various methods such as cognitive therapy, selfmonitoring, bio-feedback, compassion-focused therapy, music therapy, virtual reality assessment, language re-learning for people with post-encephalitic deficits, therapy focused on problem-solving to enhance executive functioning, interpersonal problem-solving behaviors in naturalistic simulations and several others [41]. Neurorehabilitation leads to improvements in coping skills, offering an opportunity for greater independence in everyday life. Cognitive restrictions may gradually be lifted along with the development of social skills, improved awareness and insight, increased motivation and the ability to make decisions [42]. Careful psychiatric management is indispensable for the identification, assessment and pharmacotherapy of anxiety, depression, psychosis and chronic cognitive impairment [42]. Patients and their families could be given information about voluntary-sector partners such as the Encephalitis Society (ES) or Encephalitis Global. These worldwide organizations are not only sources of support and information, but also are centers for sharing treatment, where survivors can communicate and aid each other to achieve optimal recovery [43]. They reinforce the efforts of researchers and medical institutions in partnership with the survivor community [43].

\section{Summary}

The pattern of cerebral damage inflicted in the course of viral encephalitis, which may be focal, multifocal or diffuse, usually corresponds to the acute and chronic neurological and psychiatric clinical manifestations present upon examination of the infected patient [3]. Strong headache, fever, impairment of consciousness, confusion, disorders of speech and behavior as well as general and focal neurological signs constitute the set of typical initial encephalitic symptoms $[4,44]$. It is, however, important to remember that the symptomatology present at the onset may lack neurological signs and impairment of consciousness. Caroff et al. reported delusions (54\% persecutory and nihilistic), hallucinations (auditory 44\%, visual 13\%, olfactory $3 \%)$ and affective disorders (27\%) as the initial symptoms [44]. Although the available treatment has contributed to a significant increase in the survival rates, the quality of life of encephalitis survivors remains far from satisfactory [12]. The rapid development of MRI techniques has greatly contributed to the efficacy of non-invasive diagnostics, while PCR has enabled the detection of viral genomes in the CSF [13], which is of particular significance in the case of HSE. Nevertheless, due to the rapid cerebral replication of the virus in the absence of antivirals, treatment must be initiated before the PCR results are available [19]. Besides being well tolerated, acyclovir has been proven effective experimentally and in clinical trials as well $[3,17]$. Unfortunately, the brain pathology is usually very advanced by the time a diagnosis of HSE is suspected, presumably due to the unspecific or atypical character of the symptoms [19]. What is more, the focal neurological deficits, seizures and also generalized neurological, behavioral and personality disorders may persist after the disease. The ES carried out a study among its members based on their mental self-assessment. Feelings of frustration and anger were reported by $68 \%$ of the respondents, anxiety by $67 \%$, mood swings by $59 \%$ and depression by $58 \%$ [45]. Similarly, the results of a study by Pewter et al. showed that cognitive impairments were highly prevalent and included a wide range of disorders, from severe generalized executive function deficits to isolated ones. Abnormally high levels of distress and conditions such as obsessive-compulsive disorder (OCD), depression, interpersonal sensitivity and phobias were of particularly high incidence among the participants. The study was conducted among members of the Encephalitis Society and consisted of a psychiatric examination and detailed neuropsychological testing. The authors also confirmed a correlation between depression, interpersonal anxiety and specific cognitive deficits. Such a correlation was absent with regard to OCD and phobias [4]. It is worth considering that behavioral disturbances as a component of residual symptoms often remain underestimated as causes of disability and 
distress, not only for the patients but also their families [3]. Unlike the acute phases of encephalitis, the neuropsychological and psychiatric complications remain poorly investigated and authors consistentently emphasize the urgent need for further research [22]. A more accurate comparison of outcome profiles of various encephalitis etiologies would be possible if a standardized set of neuropsychological measurements were used in the evaluation of cognitive functions in different studies [4].

Mental disorders occurring in the course of diseases associated with infections, often initially isolated and non-characteristic, may cause diagnostic difficulties. Such disorders tend to be less responsive to psychiatric management, and patients often show reduced tolerance to such treatment [5]. When conditions like an atypical course, no psychiatric treatment in the past and a negative family history occur, the specialists' attention should be directed towards mental disorders of an organic etiology $[4,16,30]$. Among possible causes like trauma, neoplasm and vascular changes, inflammatory changes of the brain as a result of a viral infection should also be considered.

The clinical pictures of the psychopathological manifestations of encephalitis that have been described in this article often have certain characteristic features that indicate the etiology. HSE is frequently characterized by a dynamic onset of symptoms with prominent consciousness disorders, epileptic seizures and symptoms of frontoorbital and temporal cortex focal damage. The intellect, on the other hand, is usually unimpaired $[5,12,17,19]$ Damage to the cerebellum and the motor cortex manifesting as ataxia with limb paresis is most indicative of TBE, while coma and seizures are not a frequent occurrence $[8,26,27]$. Although HZE may involve various brain pathologies depending on the patient's immune system, the onset is usually rather gradual $[7,33]$. Subcortical dysfunctions with retained higher nervous functions suggest herpes zoster etiology [7].

The psychotic symptoms that (especially in the acute phase) are a characteristic feature of HSE and HZE often cause diagnostic difficulties [5, 7, 19]. Encephalitis, regardless of its etiology, is likely to inflict long-lasting cognitive impairment (memory and concentration dysfunctions in particular) [22].

Differential diagnostics of encephalitis must include a thorough examination of the patient's skin because characteristic skin eruptions may be or may have been present in HSE and HZE, whereas a tick-bite inquiry should be conducted upon suspicion of TBE $[15,26,34]$. It is also worth noticing that the pathology of HZE is associated with vasculopathy, while the HSV and TBEV inflict neuronal damage directly [19]. MRI scans in HSE often reveal fronto-temporal damage, whereas hemorrhagic and ischemic lesions are a characteristic occurrence in HZE $[5,36]$. A CSF PCR study is the most conclusive procedure in diagnosing the etiology of various types of encephalitis [2]. It is a significant fact that VZV and TBEV infections may be effectively prevented by vaccination [1]. There is still no such possibility in the case of HSV, although a vaccine is the subject of current research [46].

\section{References}

[1] Parda N, Polkowska A: Zapalenia opon mózgowo-rdzeniowych i zapalenia mózgu w Polsce w 2010 roku. Przegl Epidemiol 2012, 66, 221-228.

[2] Granerod J, Crowcroft NS: The epidemiology of acute encephalitis. Neuropsychol Rehabil 2007, 17, 406-428.

[3] Arciniegas DB, Anderson CA: Viral encephalitis: neuropsychiatric and neurobehavioral aspects. Curr Psychiatry Rep 2004, 6, 372-379.

[4] Pewter SM, Williams WH, Haslam C, Kay JM: Neuropsychological and psychiatric profiles in acute encephalitis in adults. Neuropsychol Rehabil 2007, 17, 478-505.

[5] David AS, Fleminger S, Kopelman MD, Lovestone S, Mellers JDC: Lishman's Organic Psychiatry: A Textbook of Neuropsychiatry. Willey Blackwell, Oxford 2012, $4^{\text {th }}$ ed., 434-438.

[6] Mumenthaler M, Mattle H: Neurology. Urban \& Partner, Wrocław 2001, $3^{\text {rd }}$ ed., 199-201.

[7] Hokkanen L, Launes J: Cognitive outcome in acute sporadic encephalitis. Neuropsychol Rev 2000, 10, $151-167$.

[8] Kaiser R: Tick-borne encephalitis. Infect Dis Clin North Am 2008, 22, 561-575.

[9] Śpila B, Grzywa A, Helon B, Mikuła R: Paranoid syndrome and herpes simplex encephalitis - coincidence or casual nexus? Ann UMCS Sect D 2003, 58, 47-49.

[10] Zakrzewska B, Rowińska-Marcińska K, Kwieciński H, Guzek A, Strzelecki R, Przyjałkowski W, Lipowski D: Diagnostic difficulties in acute encephalitis with concurrent psychiatric disorders. Neur Neurochir Pol 1989, 23, 363-367.

[11] Koskiniemi M, Rantalaiho T, Piiparinen H, von BonsdorffCH, Fakkila M, Jarvinen A, Koskiniemi S, Kinnunen E, Mannonen L, Muttilainen M, Linnavuory K, Porras J, Puolakkainen M, Raiha K, Salonen E, Ukkonen P, Vaheri A, Valtonen V: Infections of the central nervous system of suspected viral origin: a collaborative study from Finland. J Neurovirol 2001, 7, 400-408.

[12] Steiner I: Herpes simplex virus encephalitis: new infection or reactivation? Curr Opin Neurol 2011, 24, $268-274$. 
[13] Meyding-Lamadé U, Strank C: Herpesvirus infections of the central nervous system in immunocompromised patients. Ther Adv Neurol Disord 2012, 5, 279-296.

[14] Helon B, Mikuła R: Paranoid psychosis in the course of viral encephalitis - case study. Psychiatr Pol 2003, 37, 857-862.

[15] Kennedy P, Chaudhuri A: Herpes simplex encephalitis. J Neurol Neurosurg Psychiatry 2002, 73, $237-223$.

[16] Telichowska A, Cedro A: Paranoid-catatonic syndrome in the course of viral encephalitis of Herpes Simplex etiology - a case with a favorable course. Wiad Psychiatr 2005, 8, 259-262.

[17] Steiner I, Kennedy PG, Pachner AR: The neurotropic herpes viruses: herpes simplex and varicella-zoster. Lancet Neurol 2007, 6, 1015-1028.

[18] Ghaziuddin M, Al-Khouri I, Ghaziuddin N: Autistic symptoms following herpes encephalitis. Eur Child Adolesc Psychiatry 2002, 11, 142-146.

[19] Baringer JR: Herpes simplex infections of the nervous system. Neurol Clin 2008, 26, 657-674.

[20] Lachut K, Ziółkowska-Kochan M, Kucharski R, Joachim K, Nowak M: Symptoms resembling frontotemporal dementia after an encephalitis of a probably herpes virus etiology - case study. Fam Med Primary Care Rev 2010, $12,1099-1102$.

[21] Skoldenberg B: Herpes simplex encephalitis. Scand J Infect Dis Suppl 1996, 100, 8-13.

[22] Hokkanen L, Launes J: Neuropsychological sequelae of acute-onset sporadic viral encephalitis. Neuropsychol Rehabil 2007, 17, 450-477.

[23] Hokkanen L, Launes J: Duration of transient amnesia correlates with cognitive outcome in acute encephalitis. Neuroreport 1997, 8, 2721-2725.

[24] McGrath N, Anderson NE, Croxson MC, Powell KF: Herpes simplex encephalitis treated with acyclovir: diagnosis and long term outcome. J Neurol Neurosurg Psychiatry 1997, 63, 321-326.

[25] Itzhaki RF, Lin WR, Shang D, Wilcock GK, Faragher B, Jamieson GA: Herpes simplex virus type 1 in brain and risk of Alzheimer's disease. Lancet 1997, 349, 241-244.

[26] Kaiser R: Tick-borne encephalitis. Infect Dis Clin North Am 2008, 22, 561-575.

[27] Lindquist L, Vapalahti O: Tick-borne encephalitis. Lancet 2008, 31, 1861-1871.

[28] Walder G, Lkhamsuren E, Shagdar A, Bataa J, Batmunkh T, Orth D, Heinz FX, Danichova GA, Khasnatinov MA, Würzner R, Dierich MP: Serological evidence for tick-borne encephalitis, borreliosis, and human granulocytic anaplasmosis in Mongolia. Int J Med Microbiol 2006, 296, 69-75.

[29] Grzywa A, Karakuła H, Górecka J, Chuchura M: Delusional disorders (schizophrenia-like) in the course of tickborne encephalitis and borreliosis in a patient with type A hemophilia and post-traumatic epilepsy - diagnostic and therapeutic difficulties. Pol Merk Lek 2004, 16, 59-63.

[30] Juchnowicz D, Rudnik I, Czernikiewicz A, Zajkowska J, Pancewicz SA: Mental disorders occurring in the course of the Lyme disease and tick-borne encephalitis. Prz Epidemiol 2002, 56, Suppl.1, 37-50.

[31] Hermanowska-Szpakowicz T, Pancewicz S, Szulc A, Popławska R: Mental disorders as a consequence of tickborne encephalitis (TBE). Psychiatr Pol 1997, 31, 223-230.

[32] Fowler A, Forsman L, Eriksson M, Wiskström R: Tick-borne encephalitis carries a high risk of incomplete recovery in children. J Pediatr 2013, 163, 555-560.

[33] Bangen KJ, Delano-Wood L, Wierenga CE, Stricker NH, Hesselink JR, Bondi MW: Dementia following herpes zoster encephalitis. Clin Neuropsychol 2010, 24, 1193-1203.

[34] Amlie-Lefond C, Jubelt B: Neurologic manifestations of varicella zoster virus infections. Curr Neurol Neurosci Rep 2009, 9, 430-434.

[35] McKenna KF, Warneke LB: Encephalitis associated with herpes zoster: A case report and review. Can J Psychiatry 1992, 37, 271-273.

[36] Nagel MA, Cohrs RJ, Mahalingam R, Wellish MC, Forghani B, Schiller A, Safdieh JE, Kamenkovich E, Ostrow LW, Levy M, Greenberg B, Russman AN, Katzan I,Gardner CJ, Häusler M, Nau R, Saraya T, Wada H, Goto H, de Martino M, Ueno M, Brown WD, Terborg C, Gilden DH: The varicella zoster virus vasculopathies: clinical, CSF, imaging, and virologic features. Neurology 2008, 70, 853-860.

[37] Nagel MA, Forghani B, Mahalingam R, Wellish MC, Cohrs RJ, Russman AN, Katzan I, Lin R, Gardner CJ, Gilden DH: The value of detecting anti-VZV IgG antibody in CSF to diagnose VZV vasculopathy. Neurology 2007, 68, 1069-1073.

[38] Solomon T, Michael BD, Smith PE, Sanderson F, Davies NW, Hart IJ, Holland M, Easton A, Buckley C, Kneen R, Beeching NJ: National Encephalitis Guidelines Development and Stakeholder Groups: Management of suspected viral encephalitis in adults - Association of British Neurologists and British Infection Association National Guidelines. J Infect 2012, 64, 347-373.

[39] Solomon T, Hart IJ, Beeching NJ: Viral encephalitis: a clinician's guide. Pract Neurol 2007, 7, 288-305.

[40] Venkatesan A, Tunkel AR, Bloch KC, Lauring AS, Sejvar J, Bitnun A, Stahl JP, Mailles A, Drebot M, Rupprecht CE, Yoder J, Cope JR, Wilson MR, Whitley RJ, Sullivan J, Granerod J, Jones C, Eastwood K, Ward KN, Durrheim DN, Solbrig MV, Guo-Dong L, Glaser CA: International Encephalitis Consortium. Case Definitions, Diagnostic Algorithms, And Priorities In Encephalitis: Consensus Statement Of The International Encephalitis Consortium. Clin Infect Dis 2013, 57, 1114-1128.

[41] Wilson BA: Neuropsychological rehabilitation: State of the science. S Afr J Psychology 2013, 43, 267-277.

[42] Wilson BA: Cutting Edge' Developments in Neuropsychological Rehabilitation And Possible Future Directions. Brain Impairment 2011, 12, 33-42.

[43] Eatson A, Atkin K, Hare P: 'A light in a very dark place': The role of a voluntary organization providing support for those affected by encephalitis. Neuropsychol Rehabil 2007, 17, 638-647. 
[44] Caroff SN, Mann SC, Gliatto MF, Sullivan KA, Campbell EC: Psychiatric manifestation of acute viral encephalitis. Psychiatric Annals 2001, 31, 193-204.

[45] Dowell E, Easton A, Solomon T: Consequences of encephalitis. Malton 2000, UK: Encephalitis Society.

[46] Coleman JL, Shukla D: Recent advances in vaccine development for herpes simplex virus types I and II. Hum Vaccin Immunother 2013, 26, 9.

\section{Address for correspondence:}

Bartłomiej Stańczykiewicz

Division of Consultation Psychiatry and Neuroscience

Department of Psychiatry

Wroclaw Medical University

Pasteura 10

50-367 Wrocław

Poland

Tel.: +48717841628

E-mail: bartlomiej.stanczykiewicz@umed.wroc.pl

Conflict of interest: None declared

Received: 21.08 .2013

Revised: 5.08.2014

Accepted: 19.03.2015 